\title{
BORNOLOGICAL SPACES OF NON-ARCHIMEDEAN VALUED FUNCTIONS WITH THE POINT-OPEN TOPOLOGY
}

\author{
W. GOVAERTS ${ }^{1}$
}

\begin{abstract}
F$ denotes a nontrivially non-Archimedean valued field with rank one, $X$ an ultraregular space and $C(X, F, p)$ is the vector space $C(X, F)$ of all continuous functions from $X$ into $F$ with the topology $p$ of pointwise convergence. We show that $C(X, F, p)$ is a bornological space if and only if $X$ is a Z-replete space. Also, some results are found concerning the compact-open topology $c$ and we make a comparison with that case as studied by Bachman, Beckenstein, Narici and Warner.
\end{abstract}

Introduction. The paper is self-contained, but closely related to [1] where the reader may find an extensive bibliography. As in that work, an ultraregular space is a Hausdorff one in which the clopen (closed-and-open) sets form a base of open sets. Ultraregular spaces are also widely known as zerodimensional spaces; they coincide with the $\{0,1\}$-completely regular spaces of [2] and with the $F$-completely regular spaces since $F$ itself is ultraregular.

In [1] the $E$-repletions are obtained as completions of uniform structures and $E$ is assumed to admit a compatible, separated, complete uniform structure. We shall, however, retain the older and more general purely topological view of [2] since we shall deal with a space without natural group uniformity. If $E$ is an ultraregular space, then $X$ is $E$-replete ( $E$-compact in the terminology of [2]) iff there is no ultraregular space $Y$ that contains $X$ as a dense subspace and is such that each continuous function $f$ from $X$ into $E$ may be extended to a continuous function $f^{\prime}$ from $Y$ into $E$. Equivalently, $X$ has to be homeomorphic to a closed subspace of a product of copies of $E$.

If $\mathbf{F}$ is a topological vector space over $F$, then a subset $V$ of $\mathbf{F}$ is $F$-absolutely convex iff $a x+b y \in V$ whenever $x, y \in V$ and $|a|,|b| \leqslant 1$. F is an $F$-bornological space iff the only absolutely convex sets that absorb all bounded subsets of $\mathbf{F}$ are the absolutely convex neighborhoods of 0 .

The set $|F|=\{|a|: a \in F\}$ will be provided with a topology in which all points are discrete, except for the point 0 whose neighborhoods are the usual ones. Then $|F|$ admits a natural ultrametric, defined by

$$
d(x, y)=\left\{\begin{array}{l}
0 \quad \text { if } x=y, \\
\max \{x, y\} \text { if } x \neq y .
\end{array}\right.
$$

Received by the editors September 16, 1977 and, in revised form, March 27, 1978.

AMS (MOS) subject classifications (1970). Primary 54D35, 46E10.

Key words and phrases. Non-Archimedean valued field, ultraregular space, bornological space, Z-replete space.

l“Aspirant" of the Belgian "Nationaal Fonds voor Wetenschappelijk Onderzoek". 
$|F|$ is complete in this ultrametric and therefore realcompact since its cardinality is nonmeasurable. By Theorem 9 in [1] an ultraregular space now is $|F|$-replete iff it is Z-replete. Remark also that in [1] the notation $|F|$ is used with an entirely different meaning.

We now state the key result of this paper:

THEOREM 1. $C(X, F, p)$ is an F-bornological space if and only if $X$ is a Z-replete space.

Proof OF THE "ONLY IF" PART OF THEOREM 1. Assume that there is a point $x_{\infty}$ in $v_{|F|} X \backslash X$ where $v_{|F|} X$ denotes the $|F|$-repletion of $X$. If $f \in C(X, F)$, then $|f| \in C(X,|F|)$ so that $|f|$ may be extended to a continuous function $|f|^{e} \in C\left(v_{|F|} X,|F|\right)$. We set $B=\left\{f \in C(X, F):|f|^{e}\left(x_{\infty}\right) \leqslant 1\right\}$ and show that $B$ is an absolutely convex set in $C(X, F)$, that it absorbs all bounded sets in $C(X, F, p)$ and that it is not a neighborhood.

If $f, g \in B$ and $a, b \in F$ with $|a| \leqslant 1,|b| \leqslant 1$, then clearly $|a f+b g|^{e} \leqslant$ $\max \left(|f|^{e},|g|^{e}\right)$ in all points of $X$. Hence also $|a f+b g|^{e}\left(x_{\infty}\right) \leqslant$ $\max \left(|f|^{e}\left(x_{\infty}\right),|g|^{e}\left(x_{\infty}\right)\right) \leqslant 1$, so that $a f+b g \in B$.

Now let $V$ be bounded in $C(X, F, p)$. Choose $\lambda \in F$ such that $|\lambda|>1$. If $B$ does not absorb $V$, then for all $n \geqslant 1$ there exists an $f_{n} \in V$ with $\left|f_{n}\right|^{e}\left(x_{\infty}\right)>$ $|\lambda|^{n}$. We set

$$
U_{n}=\left\{x \in v_{|F|} X:\left|f_{n}\right|^{e}(x) \leqslant|\lambda|^{n-1}\right\} .
$$

If $x \in X$, there exists $\lambda^{\prime} \in F$ with $V \subseteq \lambda^{\prime}\{f \in C(X, F):|f(x)| \leqslant 1\}$. Hence for all $n$ we have $\left|f_{n}(x)\right| \leqslant\left|\lambda^{\prime}\right|$; so $\left|f_{n}(x)\right| \leqslant|\lambda|^{n-1}$ and $x \in U_{n}$ for $n$ sufficiently large. We conclude that $x_{\infty} \notin \cup_{n=1}^{\infty} U_{n} \supseteq X$. Now let $\left(V_{n}\right)_{n=1}^{\infty}$ be obtained via $V_{p}=\left\{x \in U_{p}: x \notin U_{i}\right.$ if $\left.i<p\right\}$. Then $\left(V_{n}\right)_{n=1}^{\infty}$ is a disjoint countable family of clopen subsets of $v_{|F|} X$ and covers $X$. If $f: X \rightarrow|F|$ is obtained by putting $f(x)=|\lambda|^{n}$ whenever $x \in V_{n} \cap X$, then $f \in C(X,|F|)$ but cannot be extended to a continuous function on the whole of $v_{|F|} X$.

If $B$ is a neighborhood, consider a finite $K \subseteq X$ and $\varepsilon>0$ so that $B \supseteq\{f \in C(X, F):|f(x)| \leqslant \varepsilon$ for all $x \in K\}$. In particular $|f|^{e}\left(x_{\infty}\right) \leqslant 1$ whenever $f(x)=0$ for all $x \in K$; this is obviously false.

Introduction to the proof of the "if" part of Theorem 1 . Let $X$ be $|F|$-replete and consider an $F$-absolutely convex subset $S$ of $C(X, F)$ that absorbs all $p$-bounded sets; we intend to prove that $S$ is a $p$-neighborhood. First some notation is introduced. If $f: X \rightarrow|F|$ is an arbitrary function, then the set $B(f)=\{g \in C(X, F):|g| \leqslant f\}$ is $p$-bounded so that there is a $\lambda \in F$ with $B(f) \subseteq \lambda S$. If $f, g \in C(X,|F|)$ and $\lambda, \lambda^{\prime} \in F$, then the following are obvious:

(1) If $f \leqslant g$, then $B(f) \subseteq B(g)$.

(2) If $|\lambda| \leqslant\left|\lambda^{\prime}\right|$ and $B(f) \subseteq \lambda S$, then $B(f) \subseteq \lambda^{\prime} S$.

(3) If $B(f) \subseteq \lambda S$, then $B\left(\left|\lambda^{\prime}\right| f\right) \subseteq \lambda^{\prime} \lambda S$.

If either $f \in F^{X}$ or $f \in|F|^{X}$ and if $K$ is a subset of $X$, then $\left.f\right|_{K}$ will denote a function equal to $f$ on $K$ and zero outside of $K$; in particular $\left.1\right|_{K}$ is the characteristic function of $K$. 
Put $\mathbb{Q}=\left\{K \subseteq X: K\right.$ is clopen and there is a $\lambda \in F \backslash\{0\}$ with $B\left(\left.1\right|_{K}\right) \&$ $\lambda S\}$. Clearly $\phi \notin \mathbb{Q}$.

Lemma 1. If $S \neq C(X, F)$, then $X \in \mathbb{Q}$.

Proof. Suppose $B(1) \subseteq \lambda S$ for all $\lambda \neq 0$. Let $f \in C(X,|F|)$ be arbitrary and set $T_{n}=\{x \in X: n \leqslant f(x)<n+1\}$ for all $n=0,1, \ldots$ Suppose $B\left(f^{2}\right) \subseteq \lambda_{0} S$; we may assume $\left|\lambda_{0}\right|>1$. For all $n \geqslant 1$ we set $W_{n}=\cup_{n^{\prime}<n} T_{n^{\prime}}$, so that

$$
B(f)=B\left(\left.f\right|_{W_{n}}\right)+B\left(\left.f\right|_{\left(x \backslash W_{n}\right)}\right) .
$$

Let $\lambda \in F \backslash\{0\}$ be arbitrary. There is an $n \geqslant 1$ with $f^{2} \geqslant|\lambda| f$ on $X \backslash W_{n}$; then we have

$$
B(f) \subseteq B\left(\left.f\right|_{W_{n}}\right)+B\left(|\lambda|^{-1} f^{2}\right) \subseteq \lambda^{-1} S+\lambda^{-1} \lambda_{0} S \subseteq \lambda^{-1} \lambda_{0} S .
$$

Hence $B(f) \subseteq S$ for all $f \in C(X,|F|)$ so that $S \supseteq C(X, F)$.

LEMma 2. If $A \in \mathbb{Q}$ and if $A$ is the countable union of the clopen sets $A_{i}$ $(i=1,2, \ldots)$ then there is an $i$ with $A_{i} \in \mathbb{Q}$.

Proof. The sets $A_{i}$ may be assumed to be disjoint. If the result is not true, then $B\left(\left.1\right|_{A_{i}}\right) \subseteq \lambda S$ for all $i$ and for $\lambda \neq 0$. Choose $\lambda_{0}$ with $\left|\lambda_{0}\right|>1$ and define $f: X \rightarrow|F|$ by

$$
\begin{aligned}
f(x) & =\left|\lambda_{0}\right|^{n} \quad \text { if } x \in A_{n}, \\
& =0 \quad \text { if } x \notin A .
\end{aligned}
$$

If $B(f) \subseteq \lambda_{1} S$, then for all $n$ we have

$$
\begin{aligned}
B\left(\left.1\right|_{A}\right) & =B\left(\left.1\right|_{A_{1}}\right)+\cdots+B\left(\left.1\right|_{A_{n}}\right)+B\left(1 \mid \cup_{i>n} A_{i}\right) \\
& \subseteq \lambda S+\cdots+\lambda S+\lambda_{0}^{-n} \lambda_{1} S .
\end{aligned}
$$

Hence $B\left(\left.1\right|_{A}\right) \subseteq \lambda S$ for all $\lambda \neq 0$, a contradiction.

LEMMA 3. There exists no infinite set of disjoint members of $\mathcal{Q}$.

Proof. Suppose that $\mathbb{Q}$ contains the disjoint members $A_{n}$ for $n=1,2, \ldots$; let $B\left(\left.1\right|_{A_{n}}\right) \& \lambda_{n} S$ with $\lambda_{n} \neq 0$. Choose $\lambda \in F$ with $|\lambda|>1$ and define $f$ from $X$ into $|F|$ by setting

$$
\begin{aligned}
f(x) & =|\lambda|^{n}\left|\lambda_{n}\right|^{-1} \quad \text { if } x \in A_{n}, \\
& =0 \quad \text { if } x \notin \cup_{n=1}^{\infty} A_{n} .
\end{aligned}
$$

If $B(f) \subseteq \lambda_{0} S$, then for all $n$

$$
B\left(\left.1\right|_{A_{n}}\right) \subseteq \lambda_{0} \lambda^{-n} \lambda_{n} S
$$

If $n$ is chosen so that $\left|\lambda_{0} \lambda^{-n}\right| \leqslant 1$, a contradiction arises.

Definition. A set $A \in \mathbb{Q}$ is called special iff it is not a disjoint union of two members of $\mathbb{Q}$.

LEMMA 4. If $S \neq C(X, F)$, then $X$ is a finite union of disjoint special sets $A_{1}, A_{2}, A_{3}, \ldots, A_{n}$. 
Proof. By Lemma 1 we have $X \in \mathbb{Q}$. Suppose there is no decomposition of $X$ as described above. Then $X$ is not special, so that there is a partition $\sigma_{1}=\left\{T_{1}^{1}, T_{2}^{1}\right\}$ of $X$ with $T_{2}^{1}, T_{2}^{1}$ in $Q$. Since at least one of these two sets is not special, there is a partition $\sigma_{2}$ of $X, \sigma_{2}=\left\{T_{1}^{2}, \ldots, T_{n(2)}^{2}\right\}$, that is subordinate to $\mathcal{T}_{1}$, with $n(2)>2$ and all $T_{i}^{2}$ belonging to $Q$ if $1 \leqslant i \leqslant n(2)$.

By induction one constructs a family $\left(\mathscr{T}_{p}\right)_{p=1}^{\infty}$ of partitions of $X$ in members of $Q$ with $\mathcal{T}_{n+1}$ subordinate to $\mathcal{T}_{n}$ for all $n$ and with $\mathcal{T}_{n}$ containing more than $n$ elements. A contradiction with Lemma 3 is now easily obtained.

Lemma 5. Let $S \neq C(X, F)$ and let $A_{1}, \ldots, A_{n}$ be as in Lemma 4. Fix $i$ with $1 \leqslant i \leqslant n$. If $f \in C(X,|F|)$, then one of the following assertions holds:

(5a) $f^{-1}\left(\left[0, \lambda[) \cap A_{i} \in \mathbb{Q}\right.\right.$ for all $\lambda \in|F| \backslash\{0\}$,

(5b) there is a unique $\lambda \in|F| \backslash\{0\}$ with $f^{-1}(\lambda) \cap A_{i} \in \mathbb{Q}$.

Proof. Assume that (5a) does not hold; there is a $\lambda_{1} \in|F| \backslash\{0\}$ with $f^{-1}\left(\left[0, \lambda_{1}[) \cap A_{i} \notin \mathbb{Q}\right.\right.$. A straightforward application of Lemma 2 shows that there is a $\lambda_{2} \in|F|, \lambda_{2}>\lambda_{1}$, with $f^{-1}\left(\left[\lambda_{1}, \lambda_{2}[) \cap A_{i} \in \mathbb{Q}\right.\right.$. Since $A_{i}$ is special, the case where $F$ has a discrete valuation now becomes easy; therefore we consider a dense valuation.

By successive application of Lemma 2 we construct a sequence

$$
\begin{gathered}
\left(\left[\lambda_{j}^{0}, \lambda_{j}^{b}[)_{j=1}^{\infty} \text { with } \lambda_{1}^{0}=\lambda_{1}, \lambda_{1}^{b}=\lambda_{2},\left|\lambda_{j}^{0}-\lambda_{j}^{b}\right| \leqslant(2 / 3)^{j-1}\left|\lambda_{2}-\lambda_{1}\right|,\right.\right. \\
f^{-1}\left(\left[\lambda_{j}^{0}, \lambda_{j}^{b}[) \cap A_{i} \in \mathbb{Q}, \quad\left[\lambda_{j}^{0}, \lambda_{j}^{b}\left[\subseteq \left[\lambda_{j^{\prime}}^{0}, \lambda_{j^{\prime}}^{b}\left[\text { if } j \geqslant j^{\prime} .\right.\right.\right.\right.\right.\right.
\end{gathered}
$$

Now $A_{i} \cap \cap_{j=1}^{\infty} f^{-1}\left(\left[\lambda_{j}^{0}, \lambda_{j}^{b}[) \neq \varnothing\right.\right.$, for otherwise $A_{i}$ is the countable union of the clopen sets $A_{i} \backslash f^{-1}\left(\left[\lambda_{j}^{0}, \lambda_{j}^{b}[)\right.\right.$ neither of which belongs to $\mathbb{Q}$; this contradicts Lemma 2. Hence there is a unique $\lambda \in|F| \cap \cap_{j=1}^{\infty}\left(\left[\lambda_{j}^{0}, \lambda_{j}^{b}[)\right.\right.$. Now put $Q_{1}=\left[\lambda_{1}^{0}, \lambda_{1}^{b}\left[\backslash\{\lambda\}\right.\right.$ and $Q_{j}=\left[\lambda_{j-1}^{0}, \lambda_{j-1}^{b}[\right.$ for $j=2,3, \ldots$ Then $A_{i}=\cup_{j=1}^{\infty}\left(A_{i} \backslash f^{-1}\left(Q_{j}\right)\right)$ so that by Lemma 2 there is a $j$ with $\left(A_{i} \backslash f^{-1}\left(Q_{j}\right)\right)$ $\in \mathbb{Q}$. This is possible only for $j=1$ so that $A_{i} \cap f^{-1}\left(\left[\lambda_{1}, \lambda_{2}[\backslash\{\lambda\}) \notin \mathbb{Q}\right.\right.$; hence $A_{i} \cap f^{-1}(\lambda) \in \mathbb{Q}$.

Lemma 6. Let $S \neq C(X, F)$ and let $A_{1}, \ldots, A_{n}$ be as in Lemma 4; fix $i$ with $1 \leqslant i \leqslant n$. There is an $a_{i}$ in $A_{i}$ with the property that $G \in \mathbb{Q}$ whenever $G$ is a clopen neighborhood of $a_{i}$.

Proof. Suppose not. Put $X^{\prime}=X \cup\left\{x_{\infty}\right\}$ with $x_{\infty} \notin X$. For an arbitrary $f$ in $C(X,|F|)$ define $f^{\prime}$ from $X^{\prime}$ into $|F|$ by

$$
\begin{aligned}
f^{\prime}\left(x_{\infty}\right) & =0 \text { if } f^{-1}\left(\left[0, \lambda[) \cap A_{i} \in \mathbb{Q} \text { for all } \lambda \neq 0,\right.\right. \\
& =\lambda \text { if } f^{-1}(\lambda) \cap A_{i} \in \mathcal{Q}, \\
f^{\prime}(x) & =f(x) \text { if } x \in X .
\end{aligned}
$$

(This definition is made possible by Lemma 5.)

If $X^{\prime}$ is provided with the weak topology induced by all such functions $f^{\prime}$, then it is easily seen to be ultraregular. It contains $X$ as a dense subspace, 
which is in contradiction with the assumption that $X$ is an $|F|$-replete space.

Proof of the "if" part of Theorem 1. Let $S \neq C(X, F)$ (otherwise the result is trivial) and consider $A_{1}, \ldots, A_{n}$ as in Lemma 4 with $a_{1}, \ldots, a_{n}$ as in Lemma 6. Choose $\lambda \in|F| \backslash\{0\}$ such that $B(1) \subseteq \lambda S$ and put $U=\{f \in$ $C(X, F):\left|f\left(a_{i}\right)\right|<|\lambda|^{-1}$ for $\left.i=1, \ldots, n\right\}$. To show that $U \subseteq S$, consider $f \in U$. For each $i$ there is a clopen $G_{i}$ with $a_{i} \in G_{i} \subseteq A_{i}$ and $|f|<|\lambda|^{-1}$ on $G_{i}$.

Then

$$
|f| \leqslant|\lambda|^{-1}\left(\left.1\right|_{G_{1}}\right)+\cdots+|\lambda|^{-1}\left(\left.1\right|_{G_{n}}\right)+|f| \cdot\left(\left.1\right|_{X \backslash \cup \cup_{i-1}^{n} G_{i}}\right) .
$$

An argument similar to the one used in Lemma 1 shows that $B(|f|$. $\left(\left.1\right|_{\left.X \backslash \cup \cup_{i-1}^{n} G_{i}\right)}\right)$ is a subset of $S$ (in fact of $\lambda^{\prime} S$ for all $\lambda^{\prime} \in F \backslash\{0\}$ ); the key is that $X \backslash \cup \cup_{i=1}^{n} G_{i} \notin \mathbb{Q}$. We then have

$$
B(|f|) \subseteq \lambda^{-1} \lambda S+\cdots+\lambda^{-1} \lambda S+S \subseteq S .
$$

In particular $f$ belongs to $S$; this completes the proof of Theorem 1 .

RemarKs. (1) From [1, Theorem 9 and Theorem 13] we infer that the notions of $F$-repleteness and $\mathrm{Z}$-repleteness are identical if $F$ has a nonmeasurable cardinal. So in all practically occurring cases $C(X, F, p)$ is bornological if and only if $X$ is $F$-replete.

(2) The first part of the proof of Theorem 1 applies as well to the compact-open topology. Hence if $C(X, F, c)$ is bornological, then $X$ is Z-replete.

(3) If $F$ is complete and indiscrete and has a nonmeasurable cardinal, then by [1, Theorem 21] $C(X, F, c)$ is bornological iff $C(X, F, p)$ is bornological. We conjecture that this result is true for all $F$. The "only if" part follows from Remark 2.

ACKNOWLEDGEMENT. The author would like to thank the referee for his valuable suggestions that helped simplify the exposition of this paper.

\section{REFERENCES}

1. G. Bachman, E. Beckenstein, L. Narici and S. Warner, Rings of continuous functions with values in a topological field, Trans. Amer. Math. Soc. 204 (1975), 91-112.

2. S. Mrówka, Further results on E-compact spaces.I, Acta Math. 120 (1968), 161-185.

Seminarie voor Hogere ANalyse, Rujsuniversiteit Gent, Krijgslaan 271 (S9), B-9000 GeNT, BeLgium

Current address: Seminarie voor Hogere Analyse, Rijksuniversiteit Gent, Gaglaan 2,B-9000 Gent, Belgium 